\title{
Estudio de Percepción acerca de la Deep Web en Estudiantes de Administración de Empresas en Cartagena de Indias - Colombia
}

\author{
Perception Study on the Deep Web in Administration Students in Cartagena - Colombia \\ ${ }^{a}$ Iván Javier Monterroza Castro ${ }^{8}$, Mónica Esther Ospino Pinedo ${ }^{b}$, Blanca Inés Espinel ${ }^{c}$
}

\begin{abstract}
aimonterrosa@tecnologicocomfenalco.edu.co, Magister en Dirección Estratégica de TI, Universidad Internacional Iberoamericana Docente investigador Programa Administración de Empresas, Fundación Universitaria Tecnológico Comfenalco. Cartagena, Colombia.

b de Ingeniería de Sistemas. Universidad de Cartagena. Cartagena, Colombia.

` bespinel@tecnologicocomfenalco.edu.co, Doctora en Ciencias de la Información Universidad Complutense de Madrid. Docente investigador Programa Administración de Empresas, Fundación Universitaria Tecnológico Comfenalco. Cartagena, Colombia.
\end{abstract}

Forma de citar: I. J. Monterroza-Castro, M.E. Ospino-Pinedo y B.I. Espinel "Estudio de Percepción acerca de la Deep Web en Estudiantes de Administración de Empresas en Cartagena de Indias - Colombia”, Rev. Saberes, Vol. 13 No 02, pp. 29 - 34, 2020

Recibido: 24/03/2020 Evaluación: 28/05/2020 Aceptado: 30/06/2020 DOI: https://doi.org/10.25213/1794-4384/1302.004

\section{Resumen}

El presente trabajo de investigación fue desarrollado en dos instituciones de educación superior de la ciudad de Cartagena. El objetivo principal fue realizar estudio de percepción acerca de la Deep Web en estudiantes de administración en Cartagena - Colombia, mediante estudio de campo y un recorrido teórico que fundamentan la investigación que permitiera proponer estrategias de buenas prácticas en el uso de la internet.

Para el desarrollo de este proyecto se utilizó investigación de tipo exploratorio con muestra probabilística a 624 estudiantes objeto de investigación. Se logró determinar el grado de conocimiento que tienen los estudiantes acerca de la Deep web. Dentro de los hallazgos se pudo evidenciar la enorme cantidad de comunidades entusiastas y académicos desarrollando nuevas aplicaciones, tecnologías y hasta filosofías para sustentar estas nuevas formas de interconectar el mundo, de manera que se pueda cambiar drásticamente la percepción de las personas involucradas en este prototipo de iniciativas loables a la que se le debe dar apoyo. Además, se concluye la necesidad de continuar investigando con una mirada más profunda a la Deep Web para crear una visión más acertada de los alcances de las acciones desarrolladas sobre el uso responsable y consiente de las redes estudiadas alrededor de su uso.

\section{Palabras Clave}

Internet, piratería informática, fraudes computarizados, etica profesional.

\begin{abstract}
This research work was developed in institutions of higher education in the city of Cartagena. The main objective of this project was to conduct a perception study on the Deep Web in administration students in Cartagena Colombia, to propose good practice strategies in the use of the internet.

For the development of this project, exploratory research with probabilistic sample was used to 624 students, to determine the degree of knowledge students have about the Deep web. The findings allowed us to evidence the enormous number of enthusiastic and academic communities who are currently developing new applications, technologies and even philosophies to support these new ways
\end{abstract}

${ }^{8}$ Autor para correspondencia: correo electrónico imonterrosa@tecnologicocomfenalco.edu.co 
of interconnecting the world, so that the perception of the people involved in this prototype can be drastically changed, which are laudable initiatives that should be supported. In addition, it was possible to conclude the need to continue researching with a deeper look at the Deep Web to create a more accurate view of the scope of the actions developed on the responsible and conscious use of the networks studied around its use.

\section{Keywords}

Internet, hacking, computer fraud, professional ethics.

\section{Introducción}

Internet se ha convertido en la mayor plataforma de expresión disponible en todo el mundo. Hoy en día se puede localizar todo tipo de material en la red, y resulta casi imposible para los diversos servidores y los gobiernos controlar todo el contenido que se publica sobre el ciberespacio (Sánchez, 2015, p.101).

La Word Wide Web (WWW) es un conjunto de protocolos que permiten la consulta remota de archivos de hipertexto y utiliza internet como medio de comunicación. (Zofio Jiménez, 2013, p.7)

La Deep web o Internet profunda es el contenido secreto de Internet que no está visible para los usuarios y que requiere vías distintas a los buscadores tradicionales para llegar a sus contenidos que no siempre son adecuados para las personas (Bautista, 2015, p.26).

\section{Revisión Literaria}

\section{Web}

Aunque los inicios de Internet se remontan a los años sesenta, no ha sido hasta los años noventa cuando, gracias a la Web, se ha extendido su uso por todo el mundo. En pocos años la Web ha evolucionado enormemente: se ha pasado de páginas sencillas, con pocas imágenes y contenidos estáticos a páginas complejas con contenidos dinámicos que provienen de bases de datos, lo que permite la creación de "aplicaciones web". De forma sencilla, una aplicación web se puede definir como un programa en la cual un usuario por medio de un navegador realiza solicitudes a una aplicación remota accesible a través de Internet (o a través de una intranet) y que recibe una respuesta que se muestra en el propio navegador. (Lujan, 2002, p.49).

\section{Deep Web}

Deep Web es aquella parte de Internet que no es accesible a los motores de búsqueda basados en enlaces como Google (Ibáñez, 2017, p.75).

Hay una parte de internet que no es accesible a los motores de búsqueda tradicionales. Es normalmente lo que se denomina "internet profundo o invisible". Se trata de todo el contenido inaccesible desde los buscadores tradicionales (Sánchez, 2015, p.101).

\section{Leyes en la web}

El proceso de mercantilización del software es, y continuará siendo, un proceso contradictorio. Los intentos por definir el software como una propiedad han hecho poco para modificar la práctica de la gente de compartir ideas y programas (Peláez, 2020, p.137).

Colombia se encuentra a la vanguardia en la protección del software, es miembro tanto de la convención universal de los derechos de autor como de la convención de Berna, tratados suscritos por más de 150 países. El gobierno nacional ha robustecido la protección a los derechos de autor mediante la aprobación de nuevas leyes y la reglamentación tanto en el ámbito interno como externo (El tiempo, 1998).

\section{4. Ética en la web}

Las tecnologías de la información y de la comunicación (TIC) están invadiendo todos los aspectos de la existencia humana y están 
planteando serios desafíos a valores sociales que se consideraban firmemente establecidos (Montuschi, 2005, p.2).

La sociedad actual, requiere sujetos responsables de sus acciones, proyectos y realizaciones, necesita una finalidad, sólo desde un contexto semejante tiene sentido la ética, por ende establece fundamentos racionales, normas de comportamiento moral. Los individuos, la sociedad y las instituciones deben combatir contra el hundimiento de la moral, la crisis de los valores, para lograr mayores beneficios un mundo mejor, capaz de enfrentar los dilemas éticos (Silva \& Espina, 2006)

\section{Metodología}

Para realizar esta investigación se utilizó el tipo de investigación exploratoria, con una población conformada por estudiantes de administración de dos reconocidas instituciones de educación superior de la ciudad de Cartagena. La muestra fue de 624 estudiantes. Para la recolección de información se empleó técnica de encuesta. Para el procesamiento de encuestas, se utilizó software estadístico, Statistical Package for the Social Sciences (SPSS 24), rotación método estadístico Varimax. Para la entrada de los datos obtenidos y el análisis del mismo a través de tablas y gráficas. Con el factor de probabilidad de 095

\section{Resultados}

Los resultados arrojados en el estudio y que a continuación se muestran describen el uso de la Deep Web por estudiantes de administración de dos instituciones universitarias de Cartagena.
Tabla No. 1. Sexo

\begin{tabular}{|rl|r|r|r|r|}
\hline & & Frecuencia & Porcentaje & \multicolumn{1}{c|}{$\begin{array}{c}\text { Porcentaje } \\
\text { válido }\end{array}$} & $\begin{array}{c}\text { Porcentaje } \\
\text { acumulado }\end{array}$ \\
\hline Válido & Hombre & 254 & 40,7 & 40,7 & 40,7 \\
& Mujer & 370 & 59,3 & 59,3 & 100,0 \\
& Total & 624 & 100,0 & 100,0 & \\
\hline
\end{tabular}

Fuente: Encuestas realizadas a estudiantes de administración de la ciudad de Cartagena.

La Tabla No.1 evidencia las encuestas que se realizaron a 624 estudiantes de dos instituciones universitarias están distribuidos en 254 Hombres y 370 Mujeres.

Grafica No 1. Conocimiento acerca de Deep Web

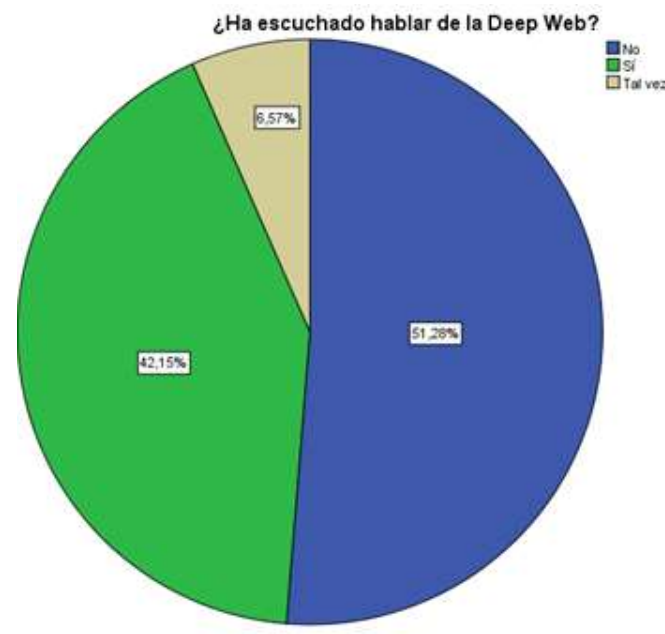

Fuente: Encuestas realizadas a estudiantes de administración de la ciudad de Cartagena.

Como se observa el 51,28\% no han escuchado hablar acerca de la Deep Web, el 42,15\% si ha escuchado hablar acerca de la Deep Web, mientras que el 6,57\% tal vez ha escuchado hablar acerca de la Deep Web. Esto significa que hay desconocimiento sobre como los estudiantes pueden usar esta herramienta que brinda a la comunidad dilucidar y construir nuevos paradigmas de sociedad, conocimiento y tecnología que aporten al proceso de desarrollo responsable y ético de estas redes, de manera que contribuyan al crecimiento de los estudiantes en su proceso de aprendizaje en la educación superior. 
Gráfica No. 2. Conocimiento acerca del contenido presente en la Deep Web

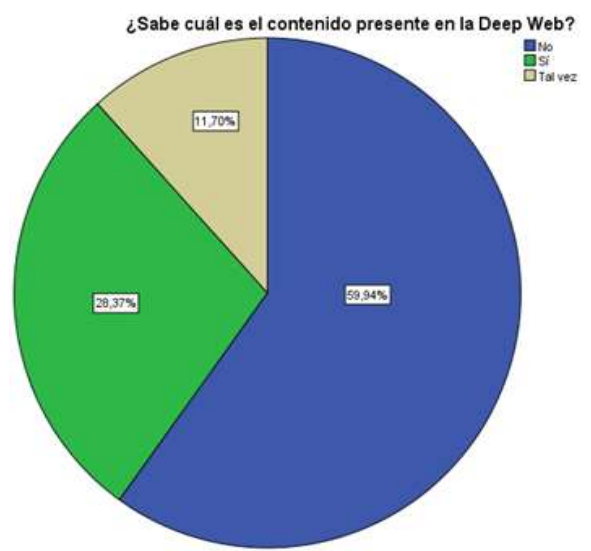

Fuente: encuestas realizadas a estudiantes de administración de la ciudad de Cartagena.

Como puede observarse el no conocimiento acerca del contenido presente en la Deep Web es $59.9 \%$, frente al $28.3 \%$ que si sabe y talvez el $11.7 \%$. cómo se puede apreciar no se aprovecha este mecanismo de la red y otras redes para aproximar al lector a su uso en diferentes plataformas, con diferentes propósitos, intercambios de archivos, servicio de mensajería de textos, correos electrónico, intercambios financieros, grupos científicos y ecosistemas sociales dispuestos para el acceso y el conocimiento del público en general, gerentes de empresas, proveedores de servicio, cuerpos de seguridad, sistemas de mercadeo para posesionar de marca entre otras.

Gráfica No. 3. Descarga de contenido con derechos de copyright sin autorización

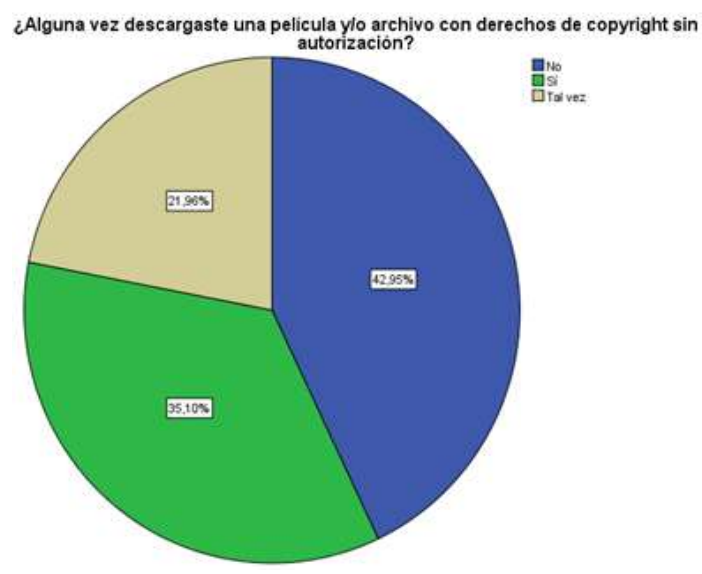

Fuente: encuestas realizadas a estudiantes de administración de la ciudad de Cartagena.
Según se observa en la gráfica anterior el $42.9 \%$ alguna vez ha descargado una película y/o archivo con derechos de copyright sin autorización; solo el $35.1 \%$ si conocen la parte legal y un $21.9 \%$ tal vez. Esto ratifica la necesidad de manejar el tema de derecho informático, pues esto podría traer múltiples conflictos alrededor del mundo se han usado redes anónimas para poder acceder a material censurado por los gobiernos por evadir los cercos informativos de regímenes políticos y para hacer saber al mundo lo que ocurre en los países con mayor control de censura informática.

Gráfica No. 4. Conciencia de violación de la ley

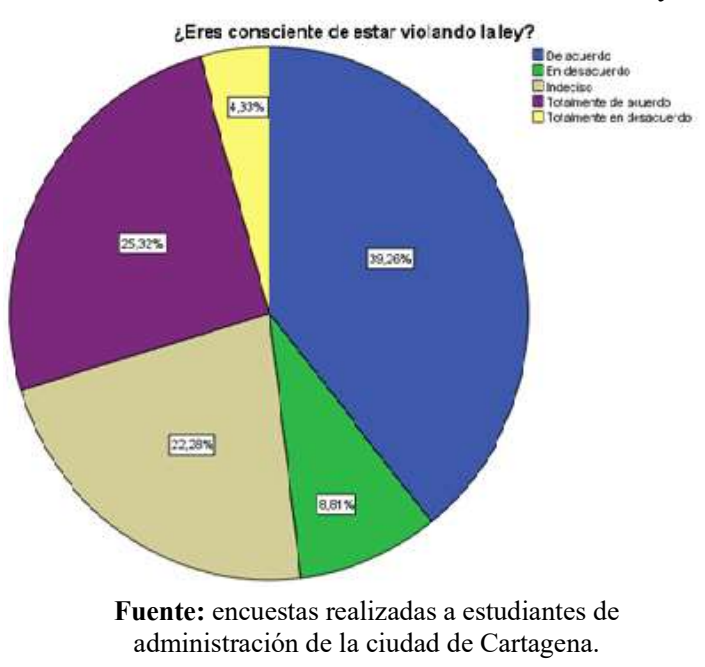

En la gráfica anterior se puede observar que frente al concepto de violación de la ley el $39.2 \%$ están de acuerdo y son conscientes de esta ilegalidad, el $25.3 \%$, están totalmente de acuerdo con la normatividad que tiene que cumplir, mientras que el $22.2 \%$, es indeciso. Esto significa la poca seguridad de conocer las normas del derecho informático, razón por la cual los gobiernos reaccionaron ante esta situación. 
Tabla No. 2. ¿Estás de acuerdo con piratería de internet?

\begin{tabular}{|c|c|c|c|c|c|}
\hline & & Frecuencia & Porcentaje & Porcentaje válido & $\begin{array}{l}\text { Porcentaje } \\
\text { acumulado }\end{array}$ \\
\hline \multirow[t]{6}{*}{ Válido } & De acuerdo & 21 & 3,4 & 3,4 & 3,4 \\
\hline & En desacuerdo & 241 & 38,6 & 38,6 & 42,0 \\
\hline & $\mathrm{Ni}$ de acuerdo ni en desacuerdo & 176 & 28,2 & 28,2 & 70,2 \\
\hline & Totalmente de acuerdo & 7 & 1,1 & 1,1 & 71,3 \\
\hline & Totalmente en desacuerdo & 179 & 28,7 & 28,7 & 100,0 \\
\hline & Total & 624 & 100,0 & 100,0 & \\
\hline
\end{tabular}

Como se puede observar en la tabla anterior sobre la piratería de Internet el $38,6 \%$, está en desacuerdo, mientras que el $28,7 \%$ dice estar totalmente en desacuerdo, el $28,2 \%$, no está $n$ i de acuerdo $n i$ en desacuerdo, lo cual significa que tienen poco conocimiento de lo que significa la piratería por Internet trayendo problemas legales.

\section{Conclusiones}

La administración educativa busca reconocer los méritos docentes, incentivar la carrera profesional, pero también crear un registro de docentes para puestos de desempeño determinados D. (Gómez \& Puello, 2018).

Internet se ha convertido en una inmensa red de redes de computadores, en la que se interconectan millones de usuarios que pueden enviar y recibir mensajes tanto de texto como de voz, consultar bases de datos desde cualquier lugar, acceder a grandes cantidades de datos e información, y al mismo tiempo establecer relaciones de manera instantánea. En este sentido, se pudo corroborar las siguientes afirmaciones:

- Se mostró el poco conocimiento de las redes más usadas y difundidas a nivel global y las distribuciones de mayor crecimiento y respaldo por la comunidad de entusiastas en la Deep Web con fines pedagógicos, de manera que sea una iniciativa loable a la que se debe seguir investigando para dar apoyo y crear una conciencia responsable acerca de su uso.

- Se evidencia la enorme cantidad de comunidades de entusiastas y académicos desarrollando procesos para innovar en aplicaciones, tecnologías y filosofías para sustentar estas nuevas formas de interconectar pares académicos quienes aportan tiempo y conocimiento que beneficien nuevas formas de optimización de aprovechar los sistemas que contribuyen a cambiar radicalmente la percepción de los estudiantes involucrados en este tipo de adquisición de iniciativas a través de conceptos específicos acerca de redes y el beneficio que les trae a maestros y estudiantes continúen en el proceso de búsqueda de conocimientos y aprendizaje. Además, encontrar nuevas prácticas pedagógicas.

- Es necesario fundamentar a los estudiantes con los conceptos teóricos sobre la piratería y violación del derecho informático como parte de la legalidad que se debe conocer con la finalidad de que se tenga claro el uso responsable y ético de las redes.

\section{Referencias Bibliográficas}

Bautista Luzardo, D. M. (2014). Deep web: aproximaciones a la ciber irresponsabilidad. Revista Latinoamericana De Bioética, 15(281), 26-37. https://doi.org/10.18359/rlbi.520

Luján Mora, S. (2002). Programación de aplicaciones web. San Vicente (Alicante): Editorial Club Universitario.

Ibáñez, E. M. (2017). Dark web y deep web como fuentes de ciberinteligencia utilizando minería de datos. $3^{\text {a }}$ Época, 74.

Montuschi, L. (2020). Cuestiones éticas problemáticas en la era de la información, internet y la world wide web. Retrieved 4 March 2020, from http://hdl.handle.net/10419/84487

Peláez, E. (2020). Software. Revista Estudios Políticos. 3(3). 35-144

Sánchez Medero, G. (2005). El ciberterrorismo: De la web 2.0 al internet profundo. Revista Ábaco, 3(85), 100-108. 
Silva, N., \&amp; Espina, (2006). Ética Informática en la Sociedad de la Información. Revista Venezolana de Gerencia, 11(36),559579

Zofio Jiménez, J. (2013). Aplicaciones web. España: Macmillan Iberia, S A.

Tiempo, C. (2020). La piratería de software y la ley. Retrieved 4 March 2020, from https://www.eltiempo.com/archivo/document o/MAM-796397

D. Gómez , J. Puello, S. Soleno-Zambrano y M. Benítez-Izquierdo, (2018). Incidencia de la Formación Pedagógica en la Práctica Docente. Revista Sostenibilidad, Tecnología y Humanismo, 9(2), 82-89. 